5. Mbindyo, P.M., et al., Developing a tool to measure health worker motivation in district hospitals in Kenya. Human resources for health, 2009. 7(1): p. 1-11.

6. Huỳnh Ngọc Tuyết Mai, Đông lực làm việc và một số yếu tố ảnh hưởng của điêu dưỡng tại các khoa lâm sàng bệnh viện Bệnh Nhiệt đới thành phố Hồ Chí Minh năm 2017, Trường Đại học Y tế công cộng. 2017.
7. Mutale, W., et al., Measuring health workers motivation in rural health facilities: baseline results from three study districts in Zambia. Human resources for health, 2013. 11(1): p. 1-8.

8. Dagne, T., W. Beyene, and N. Berhanu, Motivation and factors affecting it among health professionals in the public hospitals, Central Ethiopia. Ethiopian journal of health sciences, 2015. 25(3): p. 231-242.

\title{
TỐI ƯU HÓA CÔNG THỨC BÀO CHẾ PHIM SALBUTAMOL SULFAT GIẢI PHÓNG NHANH
}

\author{
Nguyễn Văn Bạch*, Trịnh Nam Trung*, Nguyễn Văn Minh*
}

\section{TÓM TẮT}

Mục tiêu: Bằng phần mềm tin học Modde 8.0 và INForm 3.1, tối ưu hóa được cồng thức phim salbutamol sulfat $4 \mathrm{mg}$ giải phóng nhanh. Phương pháp: Bào chế phim bằng phương pháp đổ khuôn và bốc hơi dung môi, đinh lượng salbutamol sulfat trong môi trường đo độ hòa tan bằng phương pháp quang phổ UV, thiết kế thí nghiệm và tối ưu hóa công thức bào chế phim salbutamol sulfat $4 \mathrm{mg}$ giải phóng nhanh bằng phần mềm Moddle 8.0 và INForm 3.1. Kết quả: Tỷ lệ (\%) salbutamol sulfat được giải phóng ra khỏi công thức tối ưu và số liêu dự đoán của phân mềm INForm 3.1 là tương đướng nhau (tại thời điểm 30 phút, $99,3 \%$ và $99,8 \%$ salbutamol sulfat đước giải phóng ra khỏi công thức tối ưu và số liệu của phân mềm INForm 3.1). Kết luận: Đã xây dựng được công thức tối ưu gồm có: Salbutamol sulfat 666,67mg; PVA 2,01\%; Glucose 1\%; PG 0,0201\%; Natri lauryl sulfat $0,85 \%$; Nước cất $20 \mathrm{ml}$; Ethanol $70 \%$ vừa đủ $100 \mathrm{ml}$; đổ khuôn $15 \mathrm{ml}$ vào đĩa petri đường kính $5 \mathrm{~cm}$; đường kính kính phim $10 \mathrm{~mm}$.

Tư khóa: Salbutamol sulfat, phim giải phóng nhanh.

\section{SUMMARY}

\section{FORMULATION OPTIMIZATION OF} SALBUTAMOL SULFATE FAST DISSOLVING FILM

Objective: Using Modde 8.0 and INForm 3.1 software, to optimize the fast-dissolving film formulation of salbutamol sulfate $4 \mathrm{mg}$. Methods: Preparation of films by method of casting and solvent evaporation, quantification of salbutamol sulfate in dissolution medium by UV spectroscopy, experimental design and optimization of formulations for fast dissolving film of salbutamol sulfate $4 \mathrm{mg}$ using Moddle 8.0 and INForm 3.1 software. Results: The percentage (\%) of salbutamol sulfate released from the optimal formula and the prediction data of INForm 3.1 software are similar (at 30 minutes, $99.3 \%$ and

*Hoc viên Quân Y

Chịu trách nhiệm chính: Nguyễn Văn Bạch

Email: bachhvqy@gmail.com

Ngày nhân bài: $1 / 9 / 2021$

Ngày phản biên khoa hoc: 25/9/2021

Ngày duyệt bài: 14/10/2021
$99.8 \%$ salbutamol sulfate were released from the optimal formula and data of INForm 3.1 software.). Conclusion: The optimal formula has been developed including: Salbutamol sulfate $666.67 \mathrm{mg} ;$ PVA $2.01 \%$; Glucose $1 \%$; PG $0.0201 \%$; Sodium lauryl sulfate $0.85 \%$; Distilled water $20 \mathrm{ml}$; Ethanol $70 \%$ added to $100 \mathrm{ml} ; 15 \mathrm{ml}$ were cast into a $5 \mathrm{~cm}$ diameter petri dish; films with a diameter of $10 \mathrm{~mm}$.

\section{I. ĐĂT VẤN ĐỀ}

Trong thế kỷ này, ngành công nghiệp Dược phẩm bắt đâu bị áp lực giữa giảm giá thành và chi phí gia tăng khi phát triển thuốc mới. Chi phí trung bình cho phát triển thuốc mới là khoảng 500 triệu USD trong thời gian từ 10 đến 12 năm. Trong khi cải tiến tạo ra dạng bào chế mới chỉ từ 20-50 triệu USD trong thời gian từ 3-4 năm. Khi ở dạng bào chế mới sẽ tạo ra sản phẩm có hiệu lực tốt, có khả năng cạnh tranh và mở ra các hướng điều trị mới [1]. Đường uống là đường thông dụng nhất khi sử dụng thuốc. Tuy nhiên, sẽ gặp khó khăn đối với các bệnh nhân như: Người già, trẻ em và những người gặp khó khăn khi nuốt hoặc nhai. Một nghiên cứu cho thây rằng 26\% trong số 1576 bệnh nhân đã gặp khó khăn khi nuốt viên nén. Vì vậy, hê thống giải phóng nhanh (GPN) đã ra đời vào thập kỷ 1970 để thay thế cho dạng thuốc rắn dừng đường uống [2]. Phim giải phóng nhanh là một trong những hệ thống giải phóng nhanh được sử dụng trong trường hợp bệnh nhân gặp khó khăn khi nuốt hoăc buồn nôn khi điêu trị. Khi sử dụng dược chất sẽ được hấp thu qua niêm mạc nhờ hể thống tĩnh mạch đây đặc tại niêm mạc miệng và còn được gọi là phim hòa tan tại khoang miệng. Trong nghiền cứu trước, đã khảo sát được ảnh hưởng của một số tá dược đến độ giải phóng của salbutamol từ phim giải phóng nhanh đặt tại khoang miệng [3], do đó nghiên cứu này đặt ra mục tiêu tìm được công thức tối ưu cho phim salbutamol sulfat giải phóng nhanh. 


\section{II. ĐỐI TƯỢNG VÀ PHƯƠNG PHÁP NGHIÊN CỨU \\ 2.1. Nguyên vật liệu}

- Salbutamol sulfat chuẩn: Đạt chuẩn phòng thí nghiệm (SKS: 0201042; hàm lượng: 99,50\%; độ ẩm: 0,06\%) do Viện kiểm nghiệm thuốc TW cung cấp.

- Salbutamol sulfat (Trung Quốc), PVA (Trung Quốc), PG (Ấn Độ), Glucose (Ẫn Độ), Natri lauryl sulfat (Trung Quốc), đạt tiêu chuẩn USP 38; Ethanol 70\% (Viêt Nam) đạt tiêu chuẩn DĐVN IV

\subsection{Thiết bị nghiên cứu}

- Máy thử độ hoà tan Copley DIS 8000 (Anh).

- Máy đo quang phổ UV - VIS Labomed UVD2960 (Mỹ).

- Máy khuấy cơ IKA RW16 (Hàn quốc).

- Cân phân tích Mettler toledo có độ chính xác 0,1mg (Thụy Sỹ).

- Cân kỹ thuật Sartorius độ chính xác 0,01g (Anh).

- Tủ sấy (Trung quốc).

- Đĩa petri đường kính $5 \mathrm{~cm}$ (Đức).

- Các dụng cụ khác đạt tiêu chuẩn phân tích.

\subsection{Phương pháp nghiên cứu}

2.3.1. Phương pháp nghiên cứu bào chế

Phim giải phóng nhanh được bào chế theo phương pháp đổ khuôn và bốc hơoi dung môi như sau [4], [5]:

- Dung dịch 1: Hòa tan PVA và PG trong khoảng $60 \mathrm{ml}$ ethanol $70 \%$, khuây trong 4 giờ và để yên trong 1 giờ để loại bọt khí.

- Dung dich 2: Hòa tan Salbutamol sulfat, Glucose và Natri lauryl sulfat (NaLS) trong khoảng $20 \mathrm{ml}$ nước cất.

- Phối hợp dung dịch 1 và dung dịch 2 , chuyển sang ống đong bổ sung ethanol $70 \%$ vừa đủ $100 \mathrm{ml}$, khuấy tiếp trong 1 giờ. Sau đó đổ 15 $\mathrm{ml}$ hỗn hợp vào đĩa petri đường kính $5 \mathrm{~cm}$. Sấy khô ở nhiệt độ $50^{\circ} \mathrm{C}$ trong 12 giờ trong tủ sây. Lấy phim ra cẩn thận từ đĩa petri, cắt thành hình tròn có đường kính $10 \mathrm{~mm}$ với hàm lượng dược chất đạt $4 \mathrm{mg} /$ phim. Loại bỏ những mẩu có bọt khí, rách và độ dày không đồng nhất. Sau đó, để ổn định trong bình thủy tinh đến khi đánh giá tiêu chuẩn chất lượng.

\subsubsection{Phương pháp đánh giá tiêu chuẩn}

\section{chất lượng}

Bảng 1. Các biến độc lập và khoảng biên thiên

\begin{tabular}{|c|c|c|c|c|c|}
\hline Các biến độc & Kýy & \multicolumn{5}{|c|}{ Mức biến thiên } \\
\cline { 3 - 6 } lập & hiệu & Mức trên (+1) & Mức cơ sở (0) & Mức dưới(-1) & Khoảng biến thiên \\
\hline PVA (\%) & $X 1$ & 3 & 2 & 1 & 1 \\
\hline Glucose (\%) & $X 2$ & 2 & 1,5 & 1 & 0,5 \\
\hline NaLS (\%) & $X 3$ & 1 & 0,5 & 0 & 0,5 \\
\hline
\end{tabular}

*Độ hòa tan:

+ Thiết bị: Máy cánh khuấy.

+ Tốc đọ: 50 vòng/phút.

+ Môi trường: $300 \mathrm{ml}$ dung dịch đệm phosphat pH 6,8.

+ Nhiệt độ môi trường: $37 \pm 0,5^{\circ} \mathrm{C}$.

+ Thời gian lấy mẫu: Sau các khoảng thời gian $1,3,5,10,20$ và 30 phút, hút $5 \mathrm{ml}$ môi trường hòa tan, bổ sung đồng lượng dung dịch sau mỗi lần lấy mẫu. Tiến hành định lượng bằng phương pháp quang phổ UV tại bước sóng 224nm [6].

*Định lượng bằng phương pháp UV trong quá trình đo độ hòa tan

- Khảo sát đỉnh cực đại hấp thụ của salbutamol sulfat trong mồi trường đệm phosphat pH 6,8: Pha dung dịch salbutamol sulfat có nồng độ khoảng $15 \mu \mathrm{g} / \mathrm{ml}$ trong đệm phosphat $\mathrm{pH} 6,8$, rồi tiến hành ghi phố trong vùng bước sóng từ 200-400 nm, xác định bước sóng có hấp thụ cực đại.

- Đường chuẩn Salbutamol sulfat trong môi trường đệm phosphat pH 6,8: Cân khoảng 50,5 $\mathrm{mg}$ Salbutamol sulfat bằng cân phân tích, cho vào bình định mức $50 \mathrm{ml}$, sau đó thêm dung dịch đệm phosphat $\mathrm{pH} 6,8$ vừa đủ tới vạch, lắc đều, lọc qua giấy lọc. Hút $10 \mathrm{ml}$ dịch lọc pha loãng với dung dịch đệm phosphat $\mathrm{pH} 6,8$ cho vừa đủ 100 ml. Sau đó, hút lần lượt $1 ; 1,4 ; 1,8 ; 2,2$ và 2,6 $\mathrm{ml}$ pha loãng bằng dung dịch đệm phosphat $\mathrm{pH}$ 6,8 trong bình định mức $10 \mathrm{ml}$ được các dung dịch chuẩn salbutamol sulfat có nồng độ xác định: 10,$10 ; 14,14 ; 18,18 ; 22,22$ và $26,26 \mu \mathrm{g} / \mathrm{ml}$. Đo độ hấp thụ của các dung dịch này ở bước sóng hấp thụ cực đại. Vẽ đồ thị biểu diễn mối tương quan giữa độ hấp thụ và nồng độ dung dịch. Dựa vào độ lệch chuẩn tương đối (RSD \%) và hệ số tương quan tuyến tính $\left(\mathrm{R}^{2}\right)$ để đánh giá độ lặp lại và độ tuyến tính của phương pháp.

\section{KẾT QUẢ NGHIÊN CỨU VÀ BÀN LUÂN}

\subsection{Thiết kế thí nghiệm}

Ba yếu tố là: Tỷ lệ PVA, glucose và NaLS được chon là các biến độc lập với các mức và khoảng biến thiên được xác định từ sàng lọc công thức thực nghiệm và được trình bày ở bảng 1 .

Các công thức được thiết kế nhằm lưa chọn thành phần tá dược tối ưu đế bào chế phim salbutamol sulfat GPN. Vì vậy, lựa chọn biến phụ thuộc là tỷ lệ \% dược chất giải phóng (DCGP) từ 
phim Salbutamol sulfat GPN với các tiêu chuẩn mô tả trong bảng 2.

Bảng 2. Tiêu chuẩn giải phóng dược chất của phim salbutamol sulfat GPN

\begin{tabular}{|c|c|c|}
\hline Biến phụ thuộc & Ký hiệu & Yêu câu (\%) \\
\hline \% Salbutamol giải phóng sau 1 phút & Y1 & $\geq 50$ \\
\hline \% Salbutamol giải phóng sau 5 phút & Y5 & $\geq 80$ \\
\hline \% Salbutamol giải phóng sau 30 phút & Y30 & $>90$ \\
\hline
\end{tabular}

Kết quả thiết kễ thí nghiệm: Sử dụng phần mềm Modde 8.0, thiết kế theo mô hình mặt hợp tử tại tâm được 17 công thức thực nghiệm như bảng 3.

Bảng 3. Các công thức thực nghiệm

\begin{tabular}{|c|c|c|c|c|c|c|c|}
\hline CT & PVA (\%) & Glucose (\%) & NaLS (\%) & CT & PVA (\%) & Glucose (\%) & NaLS (\%) \\
\hline N1 & 1,0 & 1,0 & 0,0 & N9 & 1,0 & 1,5 & 0,5 \\
\hline N2 & 3,0 & 1,0 & 0,0 & N10 & 3,0 & 1,5 & 0,5 \\
\hline N3 & 1,0 & 2,0 & 0,0 & N11 & 2,0 & 1,0 & 0,5 \\
\hline N4 & 3,0 & 2,0 & 0,0 & $\mathbf{N 1 2}$ & 2,0 & 2,0 & 0,5 \\
\hline N5 & 1,0 & 1,0 & 1,0 & $\mathbf{N 1 3}$ & 2,0 & 1,5 & 0,0 \\
\hline N6 & 3,0 & 1,0 & 1,0 & $\mathbf{N 1 4}$ & 2,0 & 1,5 & 1,0 \\
\hline N7 & 1,0 & 2,0 & 1,0 & $\mathbf{N 1 5}$ & 2,0 & 1,5 & 0,5 \\
\hline N8 & 3,0 & 2,0 & 1,0 & $\mathbf{N 1 6}$ & 2,0 & 1,5 & 0,5 \\
\hline & & & & $\mathbf{N 1 7}$ & 2,0 & 1,5 & 0,5 \\
\hline
\end{tabular}

3.2. Tiến hành thực nghiệm. Các thí nghiệm được tiến hành theo thứ tự ngẫu nhiên đế tránh sai số hệ thống. Sau khi bào chế xong, để phim ổn định trong 1 ngày, tiến hành định lượng Salbutamol sulfat và đo độ hòa tan. Kết quả được trình bày ở bảng 4.

Bảng 4. Độ hòa tan và hàm lương Salbutamol sulfat trong phim Salbutamol sulfat GPN ( $n=6, \bar{X} \pm S D)$

\begin{tabular}{|c|c|c|c|c|c|c|c|}
\hline \multirow{2}{*}{$\begin{array}{l}\text { Công } \\
\text { thức }\end{array}$} & \multicolumn{6}{|c|}{ Tỷ lệ (\%) Salbutamol giải phóng theo thời gian (phút) } & \multirow{2}{*}{$\begin{array}{c}\text { Hàm Iượng } \\
\text { Salbutamol(\%) }\end{array}$} \\
\hline & $\mathbf{1}$ & 3 & 5 & 10 & 20 & 30 & \\
\hline N1 & $61,3 \pm 3,1$ & $68,4 \pm 2,2$ & $76,2 \pm 3,0$ & $87,3 \pm 2,1$ & $95,4 \pm 2,4$ & $99,6 \pm 1,8$ & $98,45 \pm 1,65$ \\
\hline N2 & $10,4 \pm 0,5$ & $24,9 \pm 1,0$ & $44,2 \pm 1,1$ & $47,5 \pm 1,1$ & $59,1 \pm 0,9$ & $69,3 \pm 1,2$ & 2,38 \\
\hline N3 & $\pm 3,1$ & $71,3 \pm 2,1$ & $78,9 \pm 1,5$ & $91,2 \pm$ & & $99,1 \pm 1,5$ & \\
\hline N4 & $32,1 \pm 0,9$ & $53,5 \pm 1,2$ & $61,0 \pm 1,8$ & $77,7 \pm 1,6$ & $90,9 \pm 1,2$ & $94,0 \pm 2,2$ & 0,58 \\
\hline N5 & $72, \varepsilon$ & $80,7 \pm 2$ & $90,1 \pm 2,7$ & $97,8 \pm 1,8$ & 99, & $99,7 \pm 2,5$ & 3,51 \\
\hline N6 & & 30 & 45 & 62,1 & 1 & $73,5 \pm 2,2$ & 68 \\
\hline N7 & 2,7 & $82,1 \pm 3,2$ & $91,4 \pm 2,7$ & $98,8 \pm 2,4$ & $99,7 \pm 2,2$ & $102,1 \pm 1,8$ & $\frac{1,29}{1,29}$ \\
\hline N8 & $23,6 \pm 0,8$ & $47,4 \pm 1,3$ & $56,5 \pm 1,4$ & $73,2 \pm 1,8$ & $80,1 \pm 2,1$ & $88,7 \pm 1,9$ & $100,49 \pm 1,37$ \\
\hline N9 & $69, ?$ & 78 & $89,4 \pm 2,2$ & $95,6 \pm 2,5$ & $99,2 \pm 1,8$ & $99,8 \pm 1,2$ & \\
\hline N10 & $22,9 \pm 0,7$ & 35,1 & $54,3 \pm 1,5$ & $67,8 \pm 1,8$ & $72,7 \pm 1,6$ & $77,7 \pm 2,5$ & 3,02 \\
\hline N11 & $58,6 \pm 1,2$ & $65,3 \pm 1,5$ & $82,7 \pm 2,1$ & $91,2 \pm 2,5$ & $98,7 \pm 1,9$ & $99,6 \pm 1,2$ & $35 \pm 2,33$ \\
\hline N12 & $62,2 \pm 1,8$ & $69,7 \pm 2,1$ & $88,6 \pm 2,1$ & $96,2 \pm 1,8$ & $99,1 \pm 2,1$ & $99,8 \pm 1,4$ & $\pm 1,35$ \\
\hline & & & & & 92,6 & $99,8 \pm 1,8$ & 1,49 \\
\hline N1 & $=1,6$ & 71,2 & 90 & 97, & 99,4 & $99,9 \pm 1,6$ & $\pm 2,03$ \\
\hline N15 & $59,3 \pm 2,2$ & $68,2 \pm 2,4$ & $85,1 \pm 2,1$ & $94,8 \pm 2,5$ & $99,0 \pm 1,8$ & $99,8 \pm 1,3$ & $101,64 \pm 2,12$ \\
\hline N16 & $58,9 \pm 1,8$ & $67,6 \pm 2,0$ & $84,2 \pm 1,8$ & $93,1 \pm 1,8$ & $98,5 \pm 2,2$ & $99,6 \pm 2,3$ & $100,27 \pm 1,77$ \\
\hline N17 & $58,2 \pm 1,6$ & $67,1 \pm 2,5$ & $83,9 \pm 1,6$ & $94,3 \pm 1,6$ & $99,5 \pm 2,4$ & $101,2 \pm 2,1$ & $101,30 \pm 2,36$ \\
\hline
\end{tabular}

Nhận xét: Kết quả cho thấy hàm lượng (\%) Salbutamol sulfat của các mẫu phim trong 17 công thức thực nghiệm đạt yêu cầu về hàm lượng $(90 \%-110 \%)$. Tốc độ DCGP từ các công thức là khác nhau do tỷ lệ các loại tá dược là khác nhau. Với những công thức có tỷ lệ PVA trung bình, phim tạo ra đồng đều, trong suốt và không bị đục trong quá trình bảo quản.

\section{3. Đánh giá ảnh hưởng của các tá dược đến tốc độ giải phóng dược chất}

Thiết lập mô hình và kết quả luyện của phần mềm INForm 3.1 được thể hiện ở bảng 5 .
Bảng 5. Thiêt lâp và kết quả của mô hinh INForm

\begin{tabular}{|c|c|}
\hline \multirow{7}{*}{$\begin{array}{l}\text { Thiết lập } \\
\text { mô hình }\end{array}$} & Nhóm thứ (10\%): CT17 \\
\hline & Thông số luyện: \\
\hline & Số lớp ẩn: 1 \\
\hline & Số nút trong lớp ẩn: 2 \\
\hline & Hàm truyền: Asymmetric Sigmoid \\
\hline & Hàm truyền đâu ra: Linear \\
\hline & Thuật toán lan truyên ngược: RPROP \\
\hline \multirow{2}{*}{$\begin{array}{l}\text { Kết quả } \\
\text { mô hình }\end{array}$} & $\begin{array}{c}R^{2} \text { luyện: } Y 1=98,4393 ; Y 5=96,5286 ; \\
Y 30=99,3258\end{array}$ \\
\hline & $R^{2}$ thư: $Y 1=100 ; Y 5=100 ; Y 30=100$ \\
\hline
\end{tabular}


Nhận xét: Kết quả luyện của chương trình cho các kết quả $R^{2}$ luyện và $R^{2}$ thử nằm trong khoảng 80-100. Do đó, mô hình có sự tương quan giữa các biến độc lập và các biến phụ thuộc. Tiến hành nghiên cứu ảnh hưởng của biến độc lập lên các biến phụ thuộc qua phép phân tích mặt đáp được thể hiện ở hình 1 và 2 .

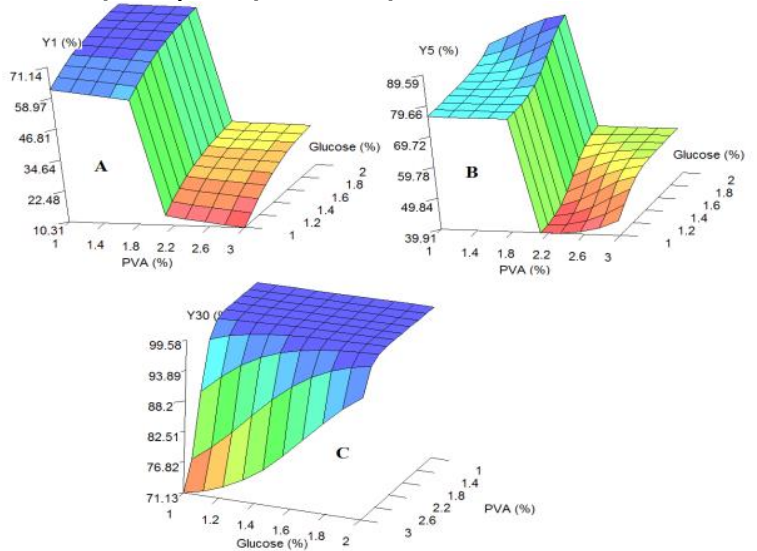

Hình 1. Mặt đáp Y1 (A), Y5 (B) và Y30 (C) theo $P V A$ và Glucose (NaLS tai tâm $=0,5 \%$ )

Nhân xét: Tại phút thứ nhất $(Y 1)$ và phút thứ 5 (Y5), khi tăng nồng độ PVA từ 1,8\% lên 2,2\% tốc độ DCGP giảm nhanh còn khi tăng tỷ lệ Glucose tốc độ DCGP tăng lên. Tại phút thứ 30 (Y30), khi nồng độ Glucose ở mức thấp, nếu tăng tỷ lệ PVA thì tốc độ DCGP giảm mạnh. Còn khi nồng độ Glucose ở mức cao, khi tăng nồng độ PVA thì tốc độ DCGP giảm nhẹ. Như vậy, PVA là polyme tạo màng có khả năng kiểm soát giải phóng còn Glucose là tá dược độn tan được trong nước có trong thành phần của phim với mục đích đảm bảo khối lượng phim cũng như tăng tốc độ DCGP.

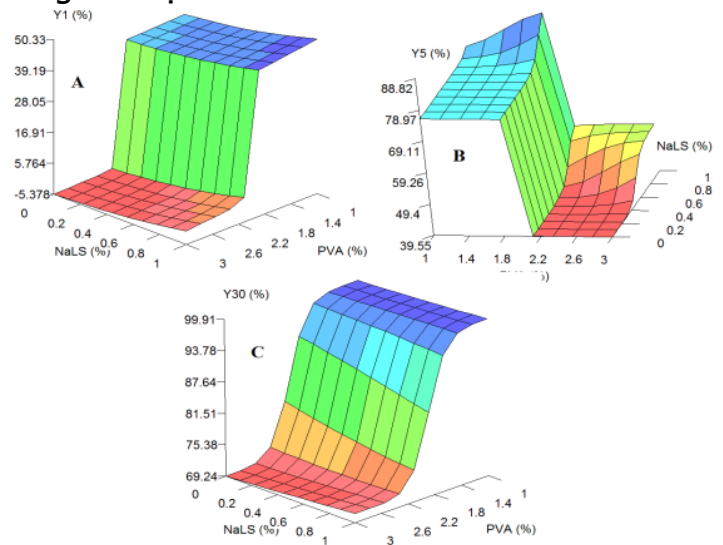

Hinh 2. Măt đáp Y1 (A), Y5 (B), Y30 (C) theo $N a L S$ và PVA (Glucose tại tâm=1,5\%)

Nhận xét: Phân tích mặt đáp nhận thẫy, tại phút thứ nhất (Y1), khi tỷ lệ NaLS tăng thì tốc độ
DCGP tăng. Tuy nhiên chủ yếu phụ thuộc vào tỷ lệ PVA. Tại phút thứ 5 (Y5), khi nồng độ PVA ở mức thấp thì NaLS ít ảnh hưởng tới mức độ DCGP. Còn khi nồng độ PVA ở mức cao, khi tăng tỉ lệ NaLS thì tốc độ DCGP cũng tăng. Tại phút thứ 30 (Y30), NaLS chỉ ảnh hưởng rõ rệt khi PVA ở tỷ lệ nồng độ từ $1,4 \%$ đến 2,6\%.

3.4. Kết quả tối ưu hóa công thức bào chế và so sánh độ hòa tan với viên đối chiếu. Từ các số liệu thực nghiệm và mục tiêu của đề tài, đă̆t ra các yêu cầu tối ưu cho các biến phụ thuộc và các kết quả đưa ra bởi phần mềm INForm 3.1 được thể hiện ở bảng 6 .

Bảng 6. Yêu cầu, kết quả và giá trị dự đoán của phần mềm INForm 3.1

\begin{tabular}{|c|c|c|c|}
\hline Yêu câu & $\mathrm{Y} 1 \geq 50 \%$ & $\mathrm{Y} 5 \geq 80 \%$ & $\mathrm{Y} 30 \geq 90 \%$ \\
\hline Kết quả tối & $\mathrm{X} 1=$ & $\mathrm{X} 2=1 \%$ & $\mathrm{X} 3=$ \\
ưu & $2,01 \%$ & & $0,85 \%$ \\
\hline Giá trị dự & $\mathrm{Y} 1=$ & $\mathrm{Y} 5=$ & $\mathrm{Y} 30=$ \\
đoán & $56,9 \%$ & $85,0 \%$ & $99,8 \%$ \\
\hline
\end{tabular}

Công thức tối ưu đưa ra bởi INForm 3.1: Salbutamol sulfat $\quad: \quad 666,67 \mathrm{mg}$ PVA $\quad: \quad 2,01 \%$ Glucose $\quad: \quad 1 \%$ PG : $\quad 0,0201 \%$ Natri lauryl sulfat $\quad: \quad 0,85 \%$ Nước cất $\quad: \quad 20 \mathrm{ml}$ Ethanol $70 \%$ vừa đủ : $\quad 100 \mathrm{ml}$ Đố khuôn $15 \mathrm{ml}$ vào đĩa petri đường kính $5 \mathrm{~cm}$ Cắt thành hình tròn đường kính $10 \mathrm{~mm}$

Quá trình giải phóng Salbutamol sulfat từ viên đối chiếu được dùng làm căn cứ để đánh giá quá trình giải phóng dược chất từ phim GPN bào chế được. Tiến hành định lượng Salbutamol sulfat trong môi trường hòa tan bằng phương pháp quang phổ UV kết quả thu được ở bảng 7 và hình 3.

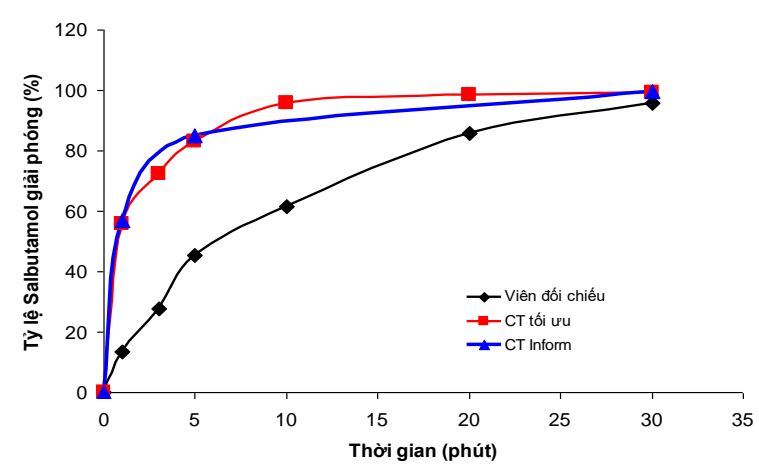

Hinh 3. Tỷ lệ (\%) Salbutamol sulfat giải phóng từ viên đôi chiếu và phim Salbutamol sulfat GPN Kết quả đánh giá độ hòa tan của công thức tối ưu và của viên đối chiếu nhận thấy: Viên đối chiếu sau 5 phút giải phóng khoảng 45,3\% dược 
chất trong khi phim Salbutamol sulfat giải phóng nhanh giải phóng được khoảng 83,2\% dược chất. Do đó, phim đã thể hiện rõ mô hình đối với dạng bào chế cần giải phóng nhanh

Bảng 7. Tỷ lệ (\%) Salbutamol sulfat giải phóng từ viên đôi chiêu và phim Salbutamol sulfat GPN $(n=6 ; \bar{X} \pm S D)$

\begin{tabular}{|c|c|c|c|c|c|c|}
\hline \multirow{2}{*}{ CT } & \multicolumn{5}{|c|}{ Tỷ lệ (\%) Salbutamol giải phóng theo thời gian (phút) } \\
\cline { 2 - 7 } & $\mathbf{1}$ & $\mathbf{3}$ & $\mathbf{5}$ & $\mathbf{1 0}$ & $\mathbf{2 0}$ & $\mathbf{3 0}$ \\
\hline Viên đối chiếu & $13,4 \pm 0,8$ & $27,8 \pm 1,2$ & $45,3 \pm 2,1$ & $61,4 \pm 2,9$ & $85,6 \pm 3,5$ & $95,8 \pm 4,2$ \\
\hline CT tối ưu & $55,9 \pm 2,9$ & $72,5 \pm 3,3$ & $83,2 \pm 4,4$ & $95,8 \pm 4,7$ & $98,4 \pm 4,8$ & $99,3 \pm 4,1$ \\
\hline Inform & 56,9 & & 85,0 & & & 99,8 \\
\hline
\end{tabular}

\section{KẾT LUẬN}

- Đã tối ưu hóa công thức phim salbutamol sulfat $4 \mathrm{mg}$ giải phóng nhanh bằng đổ khuôn và bốc hơi dung môi ở quy mô phòng thí nghiệm. Với thành phần công thức tối ưu gồm có: Salbutamol sulfat 666,67mg; PVA 2,01\%; Glucose 1\%; PG 0,0201\%; Natri lauryl sulfat 0,85\%; Nước cất 20ml; Ethanol 70\% vừa đủ 100 $\mathrm{ml}$; đổ khuôn $15 \mathrm{ml}$ vào đĩa petri đường kính $5 \mathrm{~cm}$; đường kính kính phim $10 \mathrm{~mm}$.

- Trong thử nghiệm hòa tan invitro cho thây dược chất giải phóng từ phim Salbutamol sulfat nhanh hơn so với viên đối chiếu (83,2\% so với 45,3\% sau 5 phút).

TÀI LIỆ THAM KHẢO

1. Verma R., Sanjay G. (2001), Current status of drug delivery technologies and future directions, Pharm. Technol., 25, pp. 1-14.

2. Dipika p., Upendra P., et al. (2012), Orally fast dissolving films as dominant dosage form for quick release, IJPRBS, 1(3), pp. 27- 41.

3. Nhâm Phương Thảo, Nguyễn Văn Bạch, Nguyễn Minh Chính và CS (2015), Ảnh hưởng của một số tá dược đến độ giải phóng của salbutamol từ phim giải phóng nhanh đặt tại khoang miệng, Tạp chí Y Dược học quân sự, Vol 40 (06), tr. 11-16.

4. Apoorva M., Neha C., Geeta A. (2011), Formulation and characterization of fast dissolving buccal films: A review, Der. Phar. Lettre, 3(1), pp. 152-165.

5. Mishra R., Amin A. (2005), Quick API Delivery, Pharmaceutical Technology Europe, pp. 1-5.

6. Prasanthi N. L., Sowmya K. C. et al. (2011), Design and development of sublingual fast dissolving films for an antiasthmatic drug, Der Pharmacia Lettre, 3(1), pp. 382-395.

\title{
THẨM ĐỊNH PHƯƠNG PHÁP ĐỊNH LƯợNG SALBUTAMOL SULFAT TRONG PHIM SALBUTAMOL SULFAT GIẢI PHÓNG NHANH BẰNG SẮC KÝ LỎNG HIỆU NĂNG CAO
}

\author{
Trịnh Nam Trung*, Nguyễn Văn Bạch*, Nguyễn Văn Minh*
}

\section{TÓM TẮT}

Mục tiêu: Thẩm định phương pháp định lượng salbutamol sulfat trong phim salbutamol sulfat giải phóng nhanh bằng sắc ký lỏng hiệu năng cao (HPLC) nhằm góp phần tiêu chuẩn hóa chất lượng sản phẩm. Đối tượng và phương pháp: Tiến hành định lượng salbutamol sulfat bằng phương pháp HPLC với cột sắc

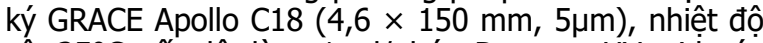
côt $25^{\circ} \mathrm{C}$, tốc đô dòng $1 \mathrm{ml} /$ phút, Detector UV tai bước sóng $276 \mathrm{~nm}$, thể tích tiêm $20 \mu \mathrm{l}$, pha động gồm dung dịch natri dihydrophosphat $\mathrm{pH} 3,1$ và methanol (85:15). Kết quả: Phương pháp đảm bảo tính thích hợp, tính đặc hiệu, khoảng tuyến tính, độ lặp lại và độ đúng theo qui định của ICH. Kết luận: Phương pháp

*Học viện Quân y

Chịu trách nhiệm chính: Trịnh Nam Trung

Email: tntqy114@gmail.com

Ngày nhận bài: $1 / 9 / 2021$

Ngày phản biên khoa học: 30/9/2021

Ngày duyệt bài: 19/10/2021 định lượng đảm bảo các yêu cầu và có thể sử dụng để định lượng salbutamol sulfat trong phim salbutamol sulfat giải phóng nhanh.

Tư khóa: Salbutamol sulfat, phim giải phóng nhanh, ICH.

\section{SUMMARY}

VALIDATION ASSAY METHOD FOR SALBUTAMOL SULFATE IN FAST DISSOLVING FILM BY HIGH PERFORMANCE LIQUID CHROMATOGRAPHY

Objective: To validate a method for the quantification of salbutamol sulfate in fast dissolving film by high performance liquid chromatography (HPLC) in order to contribute to product quality standardization. Subjects and methods: Quantitative salbutamol sulfate by HPLC method with GRACE Apollo C18 column $(4.6 \times 150 \mathrm{~mm}, 5 \mu \mathrm{m})$, column temperature $25^{\circ} \mathrm{C}$, flow rate $1 \mathrm{ml} / \mathrm{min}$, detector UV at $276 \mathrm{~nm}$, injection volume $20 \mu \mathrm{l}$, mobile phase consisting of sodium dihydrophosphate solution pH 3.1 and methanol (85:15). Results: The method ensured suitability, specificity, linearity, repeatability, 